\title{
Osteosarcoma growth suppression by riluzole delivery via iron oxide nanocage in nude mice
}

\author{
MARIAN RAGHUBIR $^{1}$, CHOWDHURY NOWSHIN RAHMAN ${ }^{1}$, JUSTIN FANG $^{2,3}$, \\ HIROSHI MATSUI $^{2-4}$ and SHAHANA SULTANA MAHAJAN ${ }^{1,5}$
}

\author{
Departments of ${ }^{1}$ Medical Laboratory Sciences, ${ }^{2}$ Chemistry, Hunter College, City University of New York, \\ New York, NY 10065; ${ }^{3}$ Department of Chemistry, The Graduate Center of City University of New York, \\ New York, NY 10016; ${ }^{4}$ Department of Biochemistry, Weill Cornell Medical College; \\ ${ }^{5}$ Brain Mind Research Institute, Weill Cornell Medical College, New York, NY 10021, USA
}

Received August 20, 2019; Accepted November 8, 2019

DOI: $10.3892 /$ or.2019.7420

\begin{abstract}
Osteosarcomas are the most commonly occurring malignant bone cancer in young individuals. The survival rate of patients with metastatic osteosarcoma is low and has been stagnant for over two decades. We previously demonstrated that the glutamate release inhibitor, riluzole inhibits osteosarcoma cell growth. Towards the development of more effective therapy, we investigated the delivery of riluzole in human metastatic osteosarcoma xenografts in mice. We compared the efficacy of riluzole delivery by intraperitoneally injecting either free riluzole or riluzole released via two different shapes of iron oxide nanoparticles (nanocage or nanosphere) of size $15 \pm 2.5 \mathrm{~nm}$. We monitored tumor size using Vernier calipers and bioluminescence assay and found a significant reduction in tumor size in the riluzole-treated groups when injected, either in free form or via nanoparticles, compared to the control groups (PBS, nanosphere or nanocage). Importantly, nanocage-delivered riluzole was most effective in reducing tumor size in the xenograft nude mice. While riluzole delivery induced apoptosis in tumor tissues in all three groups of riluzole-treated animals, it was highest in tumors from the nanocage-delivered riluzole group. Therefore, we conclude that riluzole is an effective drug to reduce tumor size in osteosarcoma and the efficacy of riluzole as a apoptotic and tumor-reducing drug is enhanced when delivered via nanocage.
\end{abstract}

\section{Introduction}

Osteosarcoma is the most common malignant bone cancer in children and young adults occurring during growth spurts $(1,2)$. At diagnosis $20 \%$ of patients present with metastatic

Correspondence to: Dr Shahana Sultana Mahajan, Department of Medical Laboratory Sciences, Hunter College, City University of New York, 425 East, 25th Street, New York, NY 10010, USA

E-mail: smahajan@hunter.cuny.edu

Key words: osteosarcoma, drug delivery, iron oxide nanoparticle, nanocage, cancer, in vivo, riluzole, xenograft osteosarcoma and $30-40 \%$ patients diagnosed with a local tumor develop metastasis later $(3,4)$. The 5 year survival rate in cases with early diagnosis is $60-75 \%$, however, in cases with metastatic disease it is approximately $30 \%(5,6)$. Unfortunately, osteosarcoma treatment outcomes for metastatic disease or recurrence have not improved in over two decades (7-9).

Cancer cells gain growth advantages over normal cells by exploiting various growth signaling pathways (10). Many cancer types also express glutamate receptors, suggesting that glutamate may play a significant role in these types of cancers $(11,12)$. Importantly, glutamate signaling is exploited by cancers of the breast, prostate and skin to enhance their growth (13-17). More recently, a genome wide association study (GWAS) found a single nucleotide alteration in the metabotropic glutamate receptor $4(M G l u R 4)$ gene in osteosarcoma patients $(17,18)$. Spontaneous secretion of glutamate and expression of glutamate receptors were demonstrated in osteosarcoma MG63 and Saos-2 cell lines (19). Since glutamate signaling plays a crucial role in tumor growth, it is crucial to investigate the mechanisms underlying glutamate signaling and to discover strategies to interrupt this signaling to prevent tumor growth.

Glutamate secretion was first shown to be prevented by a drug, riluzole, in brain slices (20). Although the mechanism of action of riluzole is not clear, it was shown to block sodium channels as well as glutamate signaling $(21,22)$. Through an unknown mechanism, riluzole was found to increase cytosolic $\mathrm{Ca}^{2+}$ levels in MG63 cells (23). Clinically used as a neuroprotectant drug in several neurological diseases such as amyotrophic lateral sclerosis (ALS) and Parkinson's disease, riluzole is currently being tested on several cancers for therapeutic purposes (24). For instance, it was found that treatment of triple-negative breast cancer cells with riluzole inhibited cell proliferation (25). In addition, riluzole was observed to reduce the growth of cancer cells in culture or in xenograft models for brain, skin, breast and prostate cancers (25-31). In a clinical trial for melanoma patients, riluzole decreased tumor size in a number of patients (32). Furthermore, in a phase II trial in patients with advanced GRM1-positive melanoma, riluzole showed some clinical benefits (33). Previously, we successfully used human metastatic osteosarcoma LM7 
cells derived from Saos-LM6 cells to study the effect of riluzole (34). We demonstrated that LM7 cells secrete glutamate and riluzole blocks secretion of glutamate, thereby inhibiting the autocrine effect on LM7 cells (28). Moreover, we demonstrated that riluzole inhibited proliferation and migration and induced apoptosis in LM7 cells. We further demonstrated that LM7 cells express metabotropic glutamate receptor, mGluR5, and knockdown of mGluR5 prevented the colony forming ability of LM7 cells (28). Thus, from many studies it is apparent that riluzole is an effective drug that inhibits cell proliferation in several types of cancers. However, the methods of delivery of riluzole which may impact the effectiveness and the outcome of riluzole therapy have not been investigated.

Nanoparticles of various natures, both organic and inorganic matter such as liposomes, peptides, cyclodextrin, viral particles, carbon nanotubes (CNTs), nano-diamonds, graphene, quantum dots, and metal-based nanoparticles, are used as theranostic agents with which to deliver cancer drugs $(35,36)$. Nanoparticle size ranges from 3 to $200 \mathrm{~nm}$; however, for escape from mononuclear phagocytosis, the size of the nanoparticle needs to be $<100 \mathrm{~nm}$. As is already known, nanoparticles with sizes of $<50 \mathrm{~nm}$ achieve better biodistribution, escape the immune system, and have improved clearance $(35,37,38)$. Small nanoparticles penetrate tumor tissue more effectively through the enhanced permeability and retention effect (EPR). Nanoparticle surface characteristics also play an important role in nanoparticle lifespan and escape from the immune system. Therefore, the surface needs to be hydrophilic, which is achieved by coating the nanoparticle surface with a hydrophilic polymer (39). Nanoparticle-mediated drug delivery improves bioavailability, enhances drug delivery, and serves as diagnostic agents $(36,40,41)$. The shape of the nanoparticles is critical to their effectiveness as a drug carrier (42-44). The shape of the nanoparticles determines the surface area to volume properties so that nanoparticles of the same size but different shapes may show different drug-loading capacity and release. Therefore, we used two iron oxide nanoparticles of the same size $(15 \pm 2.5 \mathrm{~nm})$; one a solid spherical structure (IO-sphere), and the other a cage with a hollow interior (IO-cage) offering a larger surface area and increased surface to volume ratio with higher loading and release compared to a solid IO-sphere.

We previously compared the effectiveness of free riluzole and riluzole released from nanocages or nanospheres on the apoptosis of LM7 cells in culture. We showed that riluzole released from nanoparticles is more effective in inducing apoptosis in cultured LM7 cells when compared to free riluzole (45). To determine whether this effect occurs in vivo, we tested the effect of riluzole delivery via nanospheres (IO-sphere) and nanocages (IO-cage) on osteosarcoma xenografts implanted in nude mice. Our results demonstrated that groups of nude mice injected with riluzole showed a decreased bioluminescence signal at the tumor site when compared to the control groups (PBS, nanocage, nanosphere). Moreover, the group with nanocage-delivered-riluzole showed the least intense signal. Similarly, tumor volume calculated from manual measurements demonstrated that riluzole released from the nanocages was most effective in reducing the tumor size when compared to the free riluzole or riluzole released from the nanospheres. Furthermore, tumors from the groups injected with nanocage+riluzole showed the highest percentage of apoptosis followed by the nanosphere+riluzole groups and free riluzole group. Riluzole-treated groups displayed significantly higher apoptosis compared to the control groups (PBS, nanocage or nanosphere). Thus, we showed that delivery of the drug via nanocage enhanced tumor control.

\section{Materials and methods}

Materials. 3,4-Dihydroxyhydrocinnamic acid (DHCA) (Alfa Aesar)and manganese(II) acetate,oleylamine,oleicacid,iron(II) perchlorate, and 2-(N-morpholino)ethane sulfonic acid (MES) were purchased from Sigma-Aldrich;Merck KGaA. p-Xylene, 1-ethyl-3-[3-(dimethylamino)propyl]carbodiimide (EDC) and N-hydroxysuccinimide (NHS) were purchased from Thermo Fisher Scientific, Inc. Tetrahydrofuran (THF), hexane, ferric chloride hexahydrate, phosphate-buffered saline (PBS), sodium carbonate and sodium bicarbonate were purchased from Thermo Fisher Scientific, Inc. Optimum cutting temperature compound (O.C.T. Compound) was purchased from Sakura Tech. D-luciferin was purchased from Xenogen. Riluzole was purchased from R\&D Systems (Tocris).

\section{Methods}

Iron oxide nanocage (IO-cage) synthesis. First, IO-cages were synthesized with oleic acid by a modified version of a previously published method (46). Manganese (II) acetate $(0.17 \mathrm{~g})$, oleylamine $(0.82 \mathrm{ml})$ and oleic acid $(0.16 \mathrm{ml})$ were added to p-xylene $(15 \mathrm{ml})$ in a three-necked $50 \mathrm{ml}$ flask with a reflux condenser. The flask was heated to $90^{\circ} \mathrm{C}$ in air under magnetic stirring, and then $1 \mathrm{ml}$ of deionized water was rapidly injected into the flask. The reaction mixture was heated at $90^{\circ} \mathrm{C}$ for $1.5 \mathrm{~h}$, producing $\mathrm{Mn}_{3} \mathrm{O}_{4}$ nanoparticles. One milliliter of $2.0 \mathrm{M}$ aqueous iron (II) perchlorate solution was added and the mixture was maintained at $90^{\circ} \mathrm{C}$ for an additional $1.5 \mathrm{~h}$ to produce IO-cages by galvanic replacement. After cooling, IO-cages were collected by centrifugation, rinsed with ethanol, and dispersed in an organic solvent such as hexane or THF. Then, these hydrophobic IO-cages/IO-sphere were coated with DHCA and transferred to the aqueous phase using a modified version of a previously published method (47). First, $300 \mathrm{mg}$ of DHCA was dissolved in $6 \mathrm{ml}$ of THF in a three-neck flask $(25 \mathrm{ml})$. The resulting solution was heated to $50^{\circ} \mathrm{C}$ after bubbling for $30 \mathrm{sec}$ with flowing nitrogen gas. Then, $100 \mathrm{mg}$ of hydrophobic IO-cage or IO-sphere capped by oleic acid were dispersed in $1 \mathrm{ml}$ of THF which was added dropwise to the solution. The solution was heated to $50^{\circ} \mathrm{C}$ for $3 \mathrm{~h}$, and then cooled to room temperature, and $500 \mu 1 \mathrm{NaOH}(0.5 \mathrm{M})$ was introduced to precipitate the magnetic nanoparticles. The precipitate was collected by centrifugation and resuspended in $2 \mathrm{ml}$ water, and then dialyzed overnight.

Capping of the IO-cage/IO-sphere. First, MES buffer (pH 6.0) was prepared from 0.1 M MES and $0.1 \mathrm{M} \mathrm{NaCl}$. Next, $9.6 \mathrm{mg}$ (50 $\mu \mathrm{mol})$ EDC was dissolved in $200 \mu 1$ of MES buffer (pH 6.0) and $10.9 \mathrm{mg}(50 \mu \mathrm{mol}) \mathrm{NHS}$ (N-hydroxysuccinimide) was also dissolved in $200 \mu \mathrm{l}$ of MES buffer ( $\mathrm{pH}$ 6.0). Then, to a working solution of IO-cage or IO-sphere in MES buffer (pH 6.0) was added $100 \mathrm{nmol}$ of EDC and $125 \mathrm{nmol}$ of NHS per mg of IO-cage/IO-sphere. After reacting this mixture for 
15 min, 1 mg PEG-10k-diamine per mg IO-cage/IO-sphere was dissolved and reacted in the MES buffer solution for $6 \mathrm{~h}$ while on a rocker. Then the resulting solution was dialyzed overnight with a 3,500 Da molecular weight membrane.

IO-cage/IO-sphere drug loading. Riluzole hydrochloride (25 mg) was dissolved in $\mathrm{DI}_{2} \mathrm{O}$ using serial dilutions down to $2.5 \mathrm{mg} / \mathrm{ml}$ with $40^{\circ} \mathrm{C} \mathrm{DI} \mathrm{H}_{2} \mathrm{O}$ and light rocking. Alternatively, riluzole hydrochloride could also be dissolved in DMSO, however, it is then necessary to dialyze overnight with a 3,500 Da weight membrane to remove excess DMSO from the nanoparticle solutions. Riluzole $(2.5 \mathrm{mg} / \mathrm{ml})$ was incubated in aliquots with IO-cage/sphere concentrations of $5 \mathrm{mg} / \mathrm{ml}$, and was left to shake on a rocker at $4^{\circ} \mathrm{C}$ for $6 \mathrm{~h}$. The magnetic nanoparticles containing riluzole were washed using a $1.5 \mathrm{~T}$ bar magnet to separate free riluzole hydrochloride and then subsequently resuspended in DI water. Iron contents in each aliquot of nanoparticles were quantified by UV/Vis spectroscopy (a ferric chloride peak at $351 \mathrm{~nm}$ ) after an acid digestion of iron oxide nanoparticles in $5 \mathrm{~N} \mathrm{HCl}$.

Cell culture. LM7 and LM7.eGFP.ffLuc cells (34) were obtained from Eugenie E. Kleinerman and were maintained in DMEM without glutamine supplemented with $4.5 \%$ glucose (Gibco; Thermo Fisher Scientific, Inc.) $1 \mathrm{mM}$ pyruvate (Gibco; Thermo Fisher Scientific, Inc.), 10\% fetal bovine serum (FBS) (Gibco; Thermo Fisher Scientific, Inc.), 2 mmol/1 GlutaMAX-I (Gibco; Thermo Fisher Scientific, Inc.), $100 \mathrm{U} / \mathrm{ml}$ penicillin and $100 \mu \mathrm{g} / \mathrm{ml}$ streptomycin (Gibco; Thermo Fisher Scientific, Inc.) as previously described (34). Cells were passaged every 4 days. Cells were maintained at $37^{\circ} \mathrm{C}$ in $95 \%$ air and $5 \% \mathrm{CO}_{2}$. LM7 and LM7.eGFP.ffLuc cells were tested for and were free of mycoplasma contamination.

In vivo drug delivery. Animal experiments were performed following animal protocols approved by the Institutional Animal Care and Use Committee (IACUC, protocol \#2015-0038 to Olorunsuen Ogunwobi from Hunter College) at Weill Cornell Medical College and Hunter College. Thirty-six 5 week-old NOD.Cg-Prkd $c^{\mathrm{scid}} I l 2 r g^{\mathrm{tm} 1 \mathrm{Wj} /} / \mathrm{SzJ}$ (NSG) male mice weighing $25 \mathrm{~g}$ were implanted subcutaneously with 1 million LM7.eGFP.FFLuc cells in $100 \mu$ l of PBS in the right flank. After tumors were detectable at day 5, the animals were randomly grouped into 6 groups with 6 animals in each group. The mice were treated once every day when tumors reached $200 \mathrm{~mm}^{3}$ in size, receiving daily injections of treatments via intraperitoneum (i.p.) injection for 9 days with i) PBS, or ii) riluzole or iii) neat IO-sphere or iv) IO-sphere loaded with riluzole or v) neat IO-cage, and vi) IO-cage loaded with riluzole. An injected concentration of IO-cages and IO-spheres was $75 \mu \mathrm{g} / \mathrm{kg}$, corresponding to a riluzole concentration of $2.5 \mathrm{mg} / \mathrm{kg}$.

Bioluminescence imaging. First, the animals were injected i.p. with $150 \mathrm{mg} / \mathrm{kg}$ body weight luciferin (Xenogen), and after 10 min they were anesthetized using $2 \%$ isofluorane and were imaged using the IVIS in vivo imaging system (Xenogen). Photons emitted from the luciferase-expressing LM7.eGFP. FFLuc cells in the area of the tumor in the mouse body were quantified using 'Living Image,' a software program, version 4.3.1(https://ctac.mbi.ufl.edu/files/2017/02/@-IVIS-Sp ectrum-User-Manual-4.3.1. Grayscale reference images were superimposed over the pseudocolor images, representing the emitted light intensity around the tumor site (blue least intense and red most intense). Bioluminescence imaging results were confirmed by macroscopic examination of the tumor by measurement and resection of the tumor from the euthanized animals. Animals were imaged once 2 days before they were euthanized to excise tissues $(\mathrm{N}=2$, total experimental duration=14 days).

Histological sections for TUNEL assay and hematoxylin and eosin $(H \& E)$ staining. A mixture of $10 \%$ formalin and $4 \%$ paraformaldehyde was used to fix the tumor tissues. The following day tumor tissue was incubated in a series of ethanol concentration $(70,85,95,95,100,100 \%$, respectively) and Histo-Clear (National Diagnostics), followed by three exchanges of paraffin at $60^{\circ} \mathrm{C}$ for $1 \mathrm{~h}$. Tumor tissue was sectioned and sections were deparaffinized in xylene and a series of ethanol (with high to low concentrations), followed by rehydration in deionized water prior to the TUNEL staining. The sections were permeabilized with $0.5 \%$ Triton $\mathrm{X}-100$ in PBS for 5 min followed by washing with PBS and TUNEL staining. TUNEL staining was performed as per the TUNEL staining kit instructions (Roche). Then, the sections were rinsed in three exchanges of deionized water after staining and were mounted with DAPI mounting medium. The mounted histological tumor sections were imaged multiple times using a Zeiss fluorescence microscope at $\times 20$ magnification. Ten images were obtained per tumor sample. The images were quantified by counting the number of DAPI-positive nuclei and TUNEL-positive nuclei. Tumor sections from all samples were stained using H\&E and imaged at x20 using bright fields using a Zeiss microscope.

Statistical analysis. Tumor apoptosis analysis was conducted by one way ANOVA and two different post-hoc analyses were performed by Tukey's and Bonferroni's tests. One way ANOVA was performed for the tumor volume and two different post-hoc analyses were carried out by Tukey's and Bonferroni's tests. Significance was defined at $\mathrm{P} \leq 0.05$ for the analyses.

\section{Results}

Scanning electron microscope images show comparable size of the iron oxide nanospheres and iron oxide nanocages. We performed transmission electron microscopy (TEM) of the nanoparticles (Fig. 1A and B), which showed that the IO-cages and $\mathrm{IO}$-spheres were the same size (Fig. 1A and B), using a JEOL JEM 2100). The size range of both IO-nanoparticles, IO-cage and IO-sphere was $15 \pm 2.5 \mathrm{~nm}$ and these nanoparticles were capped by polyethylene glycol (PEG) after completion of drug incorporation to yield a hydrodynamic size of $25 \pm 2.5 \mathrm{~nm}$, measured by Dynamic Light Scattering (DLS) using a Malvern Zetasizer Nano S system. Both the IO-cage and IO-sphere contained $\sim 30$ molecules of riluzole each, which was measured by assaying the riluzole concentration remaining in the supernatant. We had previously demonstrated that cellular internalization of the cage was much slower when 

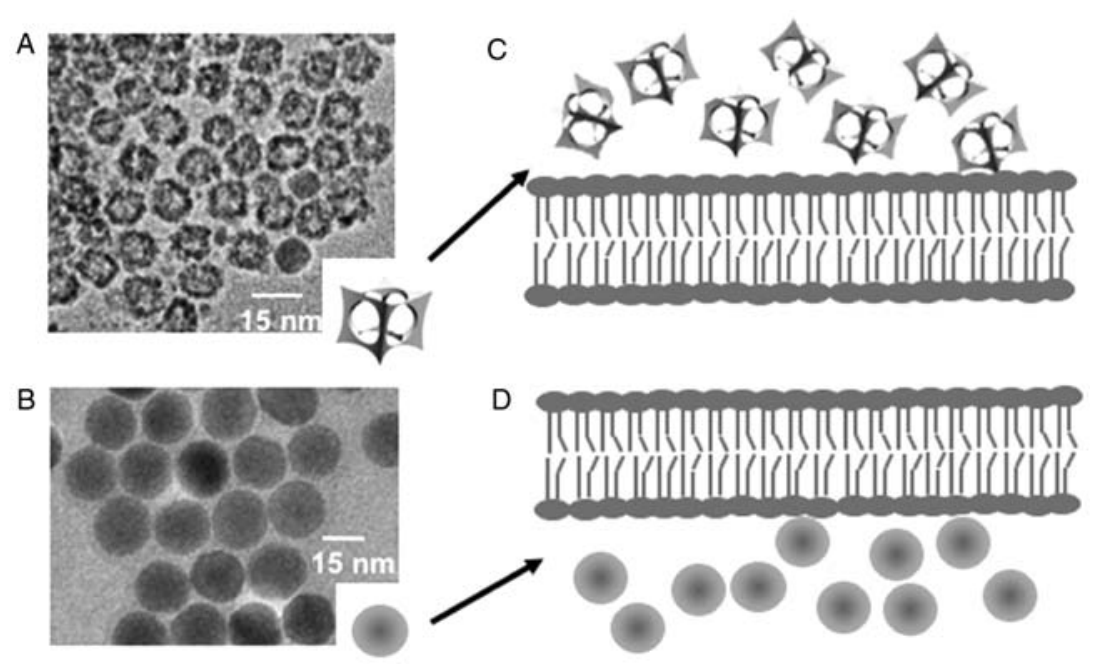

Figure 1. (A) TEM image of iron oxide nanocage (IO-cage). (B) TEM image of spherical iron oxide nanoparticles (IO-sphere). (C and D) Illustrations showing that IO-cages penetrate cell membranes much slower than IO-spheres, based on previous work. TEM, transmission electron microscopy.

compared to IO-spheres in LM7 cells in vitro (45). Slower cellular internalization of the IO-cages compared to the IO-spheres is depicted in a diagram (Fig. 1C and D).

Nanocage-delivered riluzole is most effective in tumor control. We previously demonstrated that IO-cage-delivered riluzole is more effective in inducing apoptosis in LM7 cells in vitro (45). We aimed to test the efficacy of riluzole delivery via IO-cages in reducing tumor size in a xenograft nude mouse model (protocol no. 2015-0038). For the in vivo study, we implanted one million LM7-eGFP-ff-Luc cells in 5 week-old NOD.Cg-Prkdc $c^{\text {scid }} I l 2 \mathrm{rg}^{\mathrm{tm} l \mathrm{wjl} / \mathrm{SzJ}}$ (NSG) mice in the right flank region via a subcutaneous injection. Once tumor size reached $\sim 200 \mathrm{~mm}^{3}$ on day 5 , the animals were randomly placed in 6 groups of 6 animals each and drugs were i.p. injected daily. The animals received either PBS, IO-sphere, IO-cage for controls and free riluzole, IO-sphere+riluzole or IO-cage+riluzole. We measured the tumor volume using Vernier calipers every day for 2 weeks. We calculated the tumor volume by using the formula $\left(\pi \times\right.$ length $\mathrm{x}$ width $\left.{ }^{2}\right) / 6$. The PBS group showed the largest tumors and rapid growth followed by IO-cage and IO-sphere in the control groups. Riluzole and IO-sphere+riluzole groups had significantly decreased tumor size until day 12. However, there was a slight increase in the tumor size from these groups on day 13. Samples in the control groups were not significantly different from each other. Importantly, the IO-cage-delivered riluzole group had the smallest tumor size throughout the study compared to the control groups (PBS or IO-cages alone) (Fig. 2A). We then calculated the remaining tumor volume in the groups that received riluzole either free or via IO-cage or IO-sphere. The data showed the highest tumor shrinkage in the group of mice that received riluzole via IO-cage (Fig. 2B) and was significantly different $(\mathrm{P} \leq 0.05)$ when compared to the riluzole group and IO-sphere+riluzole group.

The regression of the tumors was analyzed by bioluminescence of luciferase-expressing osteosarcoma after 12 days of the tumor implantation. We anesthetized the mice with isoflurane and i.p. injected the mice with luciferin $D$ at $150 \mathrm{mg} / \mathrm{kg}$ body weight and imaged the mice using an IVIS machine. As expected, similar to the in vitro data, riluzole released from the nanocage was found to be most effective and the mice showed the least intense signal for luciferase activity while the luciferase activity was prominent in the control groups (PBS, IO-cage alone, IO-sphere alone) (Fig. 3A). Although the riluzole and the IO-sphere+riluzole group showed a luciferase signal, the signal intensity was significantly reduced as indicated by the luminescence intensity bar (Fig. 3B and C). The control groups displayed a maximum luciferase bioluminescence at 25,000 photons per sec while the riluzole-treated groups (free riluzole, IO-sphere+riluzole or IO-cage+riluzole) showed a maximum of 4,000 photons per sec. In vivo luciferase data was corroborated by the measurement of the remaining tumor volume (Fig. 2B) in the groups of mice with riluzole treatment. These data support the outcome that riluzole delivery through the IO-cages was most effective compared to free riluzole or IO-sphere-delivered riluzole in reducing tumor size in the xenograft mouse model. Therefore, we conclude that riluzole was most effective in reducing tumor size when delivered via IO-cage.

Tumors from mice treated with nanocage-delivered riluzole exhibit increased apoptosis. Since riluzole delivery via IO-cage showed reduced tumor size, we aimed to determine the extent of apoptosis as a reason for tumor shrinkage. We performed TUNEL staining to assess the apoptosis in the tumor tissues. Tumor tissue sections were deparaffinized, fixed, permeabilized, and TUNEL assay was performed. The sections were subsequently mounted in DAPI containing mounting media and imaged using Zeiss fluorescence microscope at $x 20$. Riluzole induced apoptosis in the free riluzole, IO-sphere+riluzole and IO-cage+riluzole groups (Fig. 4A). The tumor sections stained with hematoxylin and eosin (H\&E) showed more cells in the control samples compared to fewer cells in the riluzole, IO-sphere+riluzole and IO-cage+riluzole treated sections. Analysis of DAPI-positive and TUNEL-positive cells revealed that riluzole treatment induced a higher percentage of apoptotic cells in tumor 

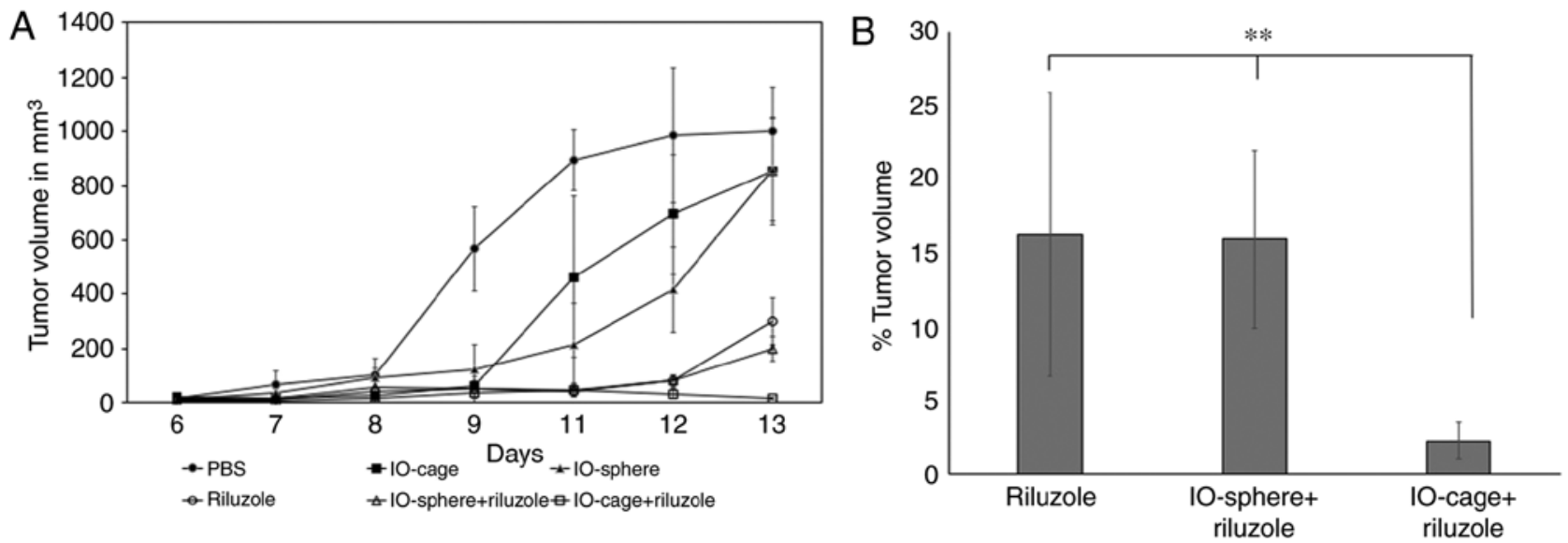

Figure 2. In vivo tumor growth volume. Tumors were measured by Vernier calipers daily once the tumors were visible. (A) The tumor volume was calculated from all 6 mice in each group. (B) Percentage of tumor volume remaining at the end of the experiment. The tumor volume results of the riluzole and IO-sphere+riluzole groups were significantly different from the IO-cage+riluzole group. ${ }^{* *} \mathrm{P} \leq 0.05$. IO, iron oxide.
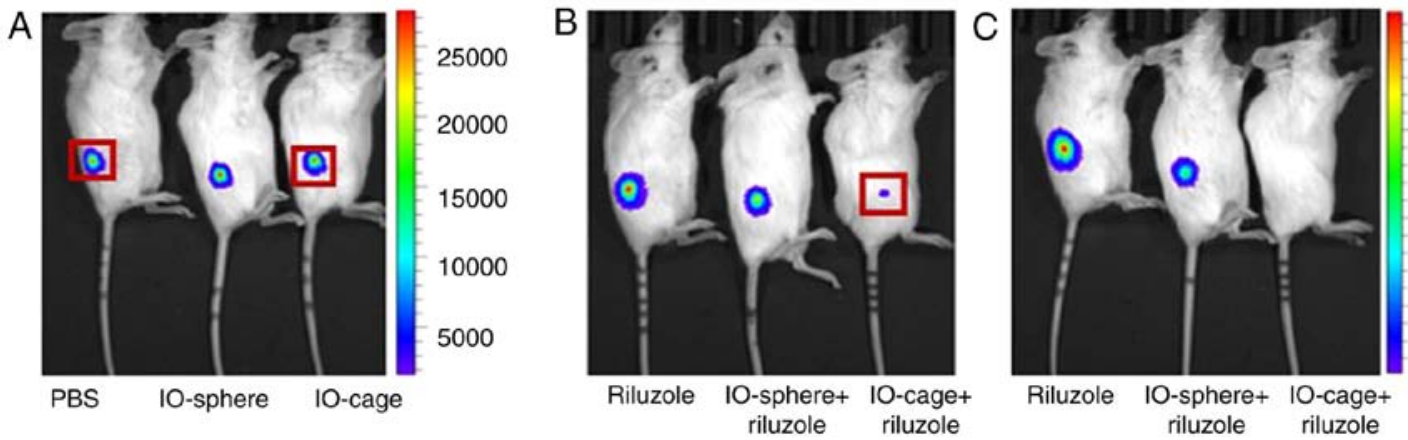

4000

3000

2000

1000

Figure 3. In vivo bioluminescence assay. Mice were injected with luciferin D, anesthetized and imaged using an IVIS machine. (A) Control mice (PBS nanosphere or nanocage). (B and C) Mice treated with riluzole in the three groups: (free riluzole, IO-sphere+riluzole and IO-cage+riluzole). Two animals in each group were analyzed by the bioluminescence assay. IO, iron oxide.

tissues of all riluzole-treated animals when compared to the control groups (PBS, IO-sphere or IO-cage) (Fig. 4B and C) with a statistically significant difference $(\mathrm{P} \leq 0.05)$. Moreover, the highest percentage of apoptosis was noted in the IO-cage+riluzole-treated group as indicated by percentage of apoptosis in the tumor tissues (Fig. 4C) and was significantly higher than that of the riluzole or IO-sphere+riluzole-treated samples $(\mathrm{P} \leq 0.05)$. Based on the apoptosis data, we found that riluzole induced apoptosis in tumors of the mice treated with riluzole (riluzole, IO-sphere+riluzole and IO-cage+riluzole groups) and the highest percentage of apoptotic cells was observed in tumors of mice when riluzole was delivered through the IO-cage.

\section{Discussion}

In the present study, we demonstrated that IO-cages and IO-spheres were of the same size and were loaded with the same number of Rilzuole molecules. We then demonstrated that LM7 cells expressing luciferase and GFP, when injected in 5 week-old nude mice formed tumors that were reduced in size by treatment with riluzole. However, the delivery of riluzole from IO-cages was most effective in shrinking tumors compared to free riluzole or riluzole released from IO-spheres, which was evident both by the in vivo bioluminescent assay and by manual measurements using Vernier calipers. Furthermore, apoptosis, measured by TUNEL assay, showed that riluzole released from IO-cages was the most effective inducer of apoptosis in tumor sections. Overall, our study demonstrated that riluzole delivery via IO-cage was more effective than free riluzole for the potential therapy for osteosarcoma.

The slower internalization of IO-cages in turn influenced drug efficacy by releasing riluzole near ion channels on membrane surfaces that alter membrane potential which subsequently inhibits glutamate release and thus prevents autocrine signaling by glutamate $(28,45)$. As described previously, the IO-cages can conceal the charges on drugs due to drug loading in the cavity, thereby highly charged drugs can be delivered using IO-cages (45).

In vivo tumor measurement using bioluminescence in living animals is an important tool for detecting and following the growth/reduction in tumor burden over time (48). We used both bioluminescence in vivo assay as well as manual measurement to assess tumor growth/shrinkage. Although our results from both methods agreed, we believe that bioluminescence assay could be used periodically at regular intervals to monitor tumor growth shrinkage and may provide accurate data. Additionally, we observed that the tumor 
A
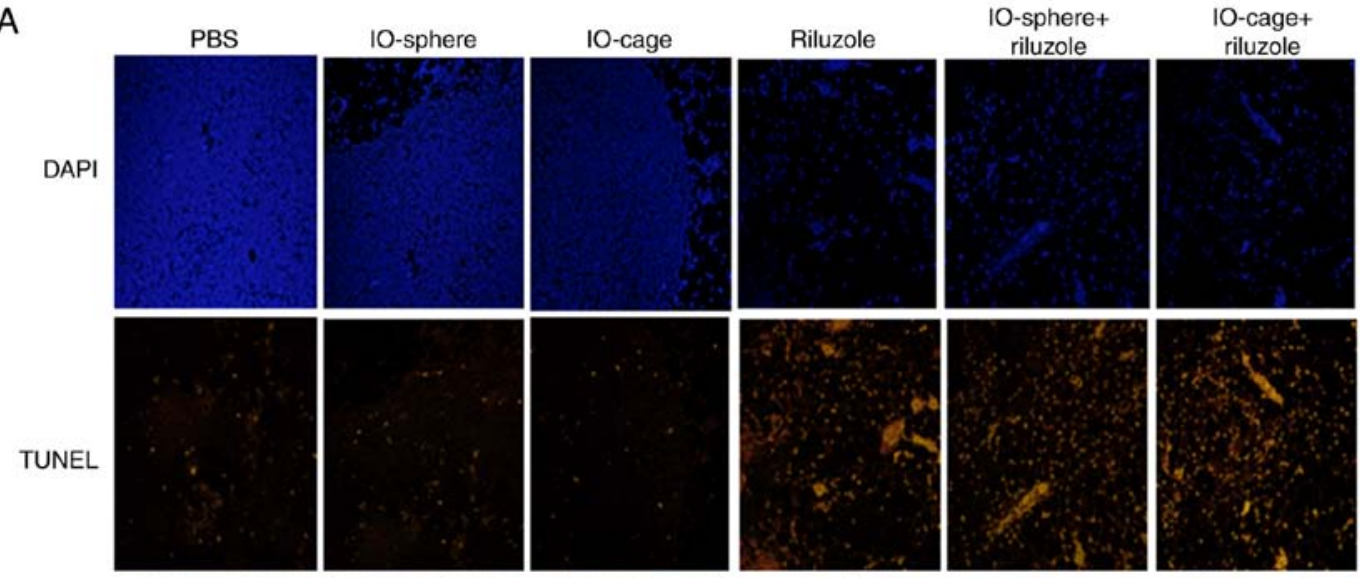

$\mathrm{H} \& \mathrm{E}$
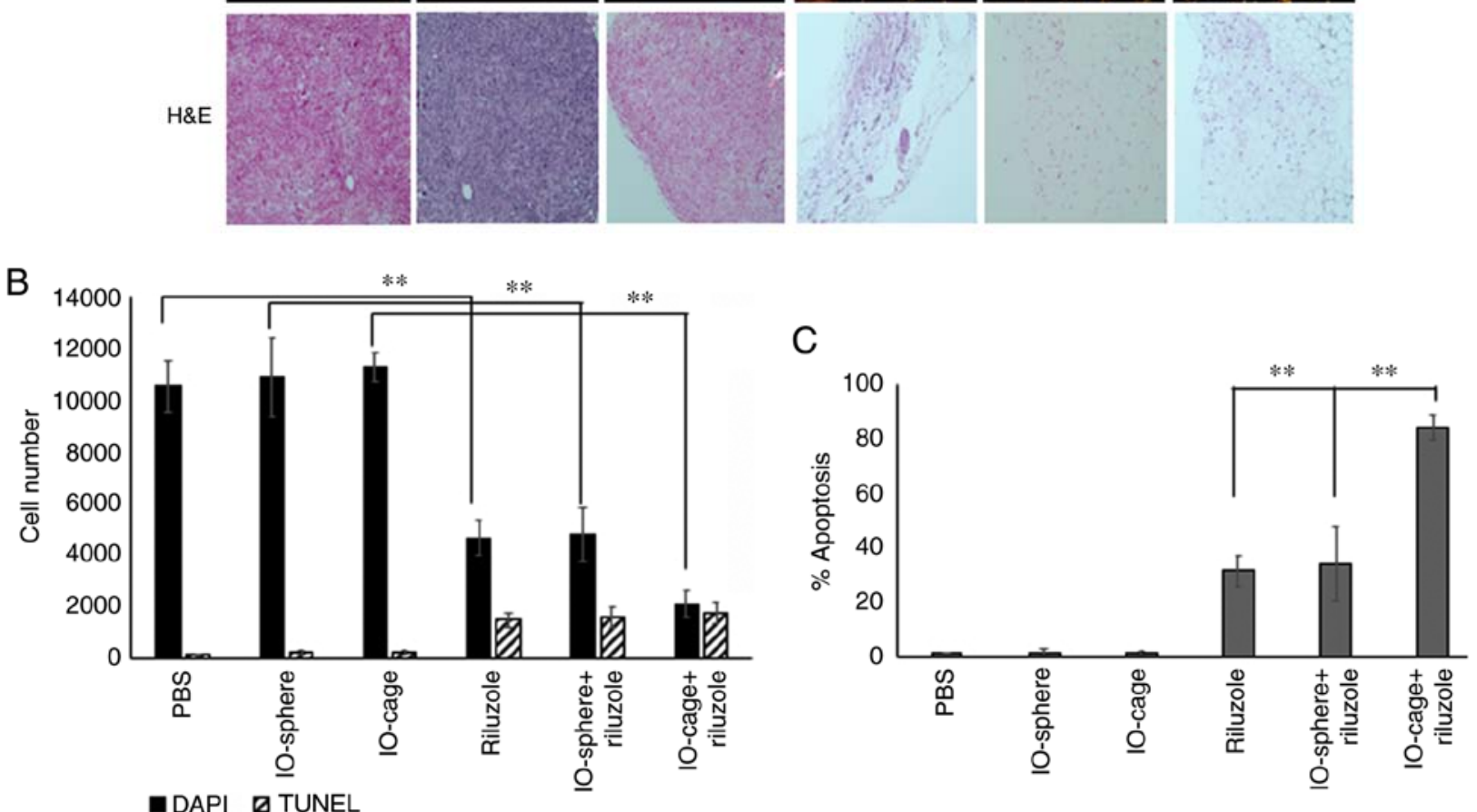

Figure 4. Tumors from the mice treated with IO-cage+riluzole show the highest apoptosis. (A) Representative images captured using fluorescence microscope at x20 magnification. Tumor sections show TUNEL-positive apoptotic nuclei in red and nuclei in blue stained with DAPI. Tumor sections were also stained with hematoxylin and eosin (H\&E). (B) Total number of DAPI-positive nuclei and apoptotic nuclei were counted from 10 images from each of the tumor sections. The controls were significantly different from the treated samples for DAPI staining (** $\mathrm{P} \leq 0.05)$. The TUNEL results for the controls were also significantly different than the treated samples $\left({ }^{* *} \mathrm{P} \leq 0.05\right)$. (C) The percentage of apoptosis in each group was calculated by using DAPI-positive nuclei and TUNEL-positive nuclei. Overall the percentage of apoptosis in the controls was significantly less than that noted in the riluzole, IO-sphere+riluzole and IO-cage+riluzole groups $\left({ }^{* *} \mathrm{P} \leq 0.05\right)$. There was a significant difference in percentage of apoptosis between the riluzole and IO-sphere+riluzole groups when compared with that of the IO-cage+riluzole group $\left({ }^{* *} \mathrm{P} \leq 0.05\right)$. IO, iron oxide.

growth shrinkage was most significant on the last day, day 13 , when compared to free riluzole or riluzole released from the IO-sphere, thus we believe that a relatively longer duration of the experiment may further discriminate the effectiveness of the cage vs. sphere as suggested by luciferase assay and apoptosis in the tumor tissue. Recent studies with gold particles with an average size of $200 \mathrm{~nm}$ have demonstrated that star-shaped and rod-shaped gold nanoparticles showed the highest cytotoxicity in osteosarcoma and pancreatic duct cell lines compared to spherical gold nanoparticles of the same size in vitro (49). Furthermore, star-shaped and rod-shaped gold nanoparticles stimulated expression of Bax protein and caused increased cytotoxicity. Therefore, the shape of the nanoparticle is crucial in determining delivery of the drug as well as inducing cytotoxicity.
The effective serum concentration of riluzole in humans determined from previous clinical trials is $\sim 50 \mathrm{mg}$ oral daily dose; the area under the curve (AUC) in serum at $24 \mathrm{~h}$ is approximately $2,000 \mathrm{ng} / \mathrm{ml}(50,51)$. We injected $50 \mu \mathrm{M}$ riluzole intraperitoneally (i.p.) in the mice daily for the in vivo experiments, which is approximately $400 \mathrm{ng} / \mathrm{ml}$ blood in mice. The serum concentration with the i.p. injected riluzole, which does not undergo hepatic clearance, was 5 times less compared to the oral dose in humans and was effective and well within the tolerated dose in humans. Riluzole released from IO-cages or IO-spheres may show differences in biodistribution and pharmacokinetics and pharmacodynamics when compared to free riluzole. This issue warrants further investigation. Interestingly, riluzole delivered via $88 \mathrm{~nm}$ liposomes for targeted delivery to the brain in rats showed lower biodistribution in other organs (52). 
In our study, the half-life of riluzole may have been prolonged due to delivery via the IO-cage where riluzole is incorporated in the cavity of the IO-cage preventing it from the hydrophilic environment until release thus prolonging the half-life of riluzole. This needs to be further investigated. Furthermore, in the present study, the tumor burden in the PBS group was too large and prevented further investigation on the effect of riluzole delivery on metastasis. Therefore, future investigations need to focus on riluzole delivery using $\mathrm{IO}$-spheres or IO-cages and the efficacy on metastasis in a metastasis model.

We conclude that the delivery of riluzole was most effective in reducing osteosarcoma tumor size in nude mice via the IO-cage when compared to free riluzole and IO-sphere-delivered riluzole. The effectiveness of riluzole may be due to the slower internalization of IO-cages and riluzole loading at a high concentration in the hollow core of the IO-cage. The small size of IO-cages may serve several advantages including evasion from the immune system, better biodistribution and delivery of a high dose of riluzole. The IO-cage-mediated delivery of riluzole may be applied to other cancer models that depend on glutamate for growth signaling or for the delivery of drugs that carry charge.

\section{Acknowledgements}

We thank Eugenie E. Kleinerman from the MD Anderson Cancer Center for the generous gift of LM7 and LM7-eGFP-ff-Luc cells. We thank Dr Olorunseun Ogunwobi for the animal protocol. We thank Dr Upal Basu-Roy for the statistical analysis of the data. We thank Dr Alka Mansukhani and Dr Muktar Mahajan for critical comments on the manuscript.

\section{Funding}

The research study was funded by PSC CUNY \#47 to Shahana S. Mahajan and the material parts were supported by the National Institute on Minority Health and Health Disparities (NIMHD) of NIH (MD007599).

\section{Availability of data and materials}

Data and material will be made available upon request.

\section{Authors' contributions}

MR carried out the in vivo experiment and analyzed the data SSM conceived and supervised the study. JF and HM organized the nanoparticle synthesis, drug loading, capping modification, and TEM imaging. CNR performed the apoptosis assay. All authors read and approved the manuscript and agree to be accountable for all aspects of the research in ensuring that the accuracy or integrity of any part of the work are appropriately investigated and resolved.

\section{Ethics approval and consent to participate}

Animal study was preapproved by the IACUC Committee at Weill Cornell Medical College, and the studies were carried out in accordance with the approved protocol. The approved protocol number is \#2015-0038.

\section{Patient consent for publication}

Not applicable.

\section{Competing interests}

The authors declare that they have no competing interest.

\section{References}

1. Dorfman HD and Czerniak B: Bone cancers. Cancer 75: 203-210, 1995.

2. Whelan JS: Osteosarcoma. Eur J Cancer 33: 1611-1618, 1997.

3. Kaste SC, Pratt CB, Cain AM, Jones-Wallace DJ and Rao BN: Metastases detected at the time of diagnosis of primary pediatric extremity osteosarcoma at diagnosis: Imaging features. Cancer 86: 1602-1608, 1999.

4. Mialou V, Philip T, Kalifa C, Perol D, Gentet JC, Marec-Berard P, Pacquement H, Chastagner P, Defaschelles AS and Hartmann O: Metastatic osteosarcoma at diagnosis: Prognostic factors and long-term outcome-the french pediatric experience. Cancer 104: 1100-1109, 2005

5. Berner K, Johannesen TB, Berner A, Haugland HK, Bjerkehagen B, Bohler PJ and Bruland OS: Time-trends on incidence and survival in a nationwide and unselected cohort of patients with skeletal osteosarcoma. Acta Oncol 54: 25-33, 2015.

6. Mirabello L, Troisi RJ and Savage SA: Osteosarcoma incidence and survival rates from 1973 to 2004: Data from the surveillance, epidemiology, and end results program. Cancer 115: 1531-1543, 2009.

7. Geller DS and Gorlick R: Osteosarcoma: A review of diagnosis, management, and treatment strategies. Clin Adv Hematol Oncol 8: 705-718, 2010.

8. Lindsey BA, Markel JE and Kleinerman ES: Osteosarcoma overview. Rheumatol Ther 4: 25-43, 2017.

9. Morrow JJ and Khanna C: Osteosarcoma genetics and epigenetics: Emerging biology and candidate therapies. Crit Rev Oncog 20: 173-197, 2015.

10. Martin GS: Cell signaling and cancer. Cancer Cell 4: 167-174, 2003.

11. Stepulak A, Luksch H, Gebhardt C, Uckermann O, Marzahn J, Sifringer M, Rzeski W, Staufner C, Brocke KS, Turski L and Ikonomidou C: Expression of glutamate receptor subunits in human cancers. Histochem Cell Biol 132: 435-445, 2009.

12. Stepulak A, Rola R, Polberg K and Ikonomidou C: Glutamate and its receptors in cancer. J Neural Transm (Vienna) 121: 933-944, 2014.

13. Koochekpour S: Glutamate, a metabolic biomarker of aggressiveness and a potential therapeutic target for prostate cancer. Asian J Androl 15: 212-213, 2013.

14. Pollock PM, Cohen-Solal K, Sood R, Namkoong J, Martino JJ, Koganti A, Zhu H, Robbins C, Makalowska I, Shin SS, et al: Melanoma mouse model implicates metabotropic glutamate signaling in melanocytic neoplasia. Nat Genet 34: 108-112, 2003.

15. Willard SS and Koochekpour S: Glutamate signaling in benign and malignant disorders: Current status, future perspectives, and therapeutic implications. Int J Biol Sci 9: 728-742, 2013.

16. Willard SS and Koochekpour S: Glutamate, glutamate receptors, and downstream signaling pathways. Int J Biol Sci 9: 948-959, 2013.

17. Yu LJ, Wall BA, Wangari-Talbot $\mathrm{J}$ and Chen S: Metabotropic glutamate receptors in cancer. Neuropharmacology 15: 193-202, 2016.

18. Savage SA, Mirabello L, Wang Z, Gastier-Foster JM, Gorlick R, Khanna C, Flanagan AM, Tirabosco R, Andrulis IL, Wunder JS, et al: Genome-Wide association study identifies two susceptibility loci for osteosarcoma. Nat Genet 45: 799-803, 2013.

19. Kalariti NP, Lembessis $P$ and Koutsilieris M: Characterization of the glutametergic system in MG-63 osteoblast-like osteosarcoma cells. Anticancer Res 24: 3923-3929, 2004.

20. Martin D, Thompson MA and Nadler JV: The neuroprotective agent riluzole inhibits release of glutamate and aspartate from slices of hippocampal area CA1. Eur J Pharmacol 250: 473-476, 1993. 
21. Doble A: The pharmacology and mechanism of action of riluzole. Neurology 47 (Suppl 4): S233-S241, 1996.

22. Hubert JP, Delumeau JC, Glowinski J, Premont J and Doble A: Antagonism by riluzole of entry of calcium evoked by NMDA and veratridine in rat cultured granule cells: Evidence for a dual mechanism of action. Br J Pharmacol 113: 261-267, 1994.

23. Jan CR, Lu YC, Jiann BP, Chang HT and Huang JK: Effect of riluzole on cytosolic $\mathrm{Ca}^{2+}$ increase in human osteosarcoma cells. Pharmacology 66: 120-127, 2002.

24. Liu J and Wang LN: The efficacy and safety of riluzole for neurodegenerative movement disorders: A systematic review with meta-analysis. Drug Deliv 25: 43-48, 2018.

25. Speyer CL, Smith JS, Banda M, DeVries JA, Mekani T and Gorski DH: Metabotropic glutamate receptor-1: A potential therapeutic target for the treatment of breast cancer. Breast Cancer Res Treat 132: 565-573, 2012.

26. Akamatsu K, Shibata MA, Ito Y, Sohma Y, Azuma H and Otsuki Y: Riluzole induces apoptotic cell death in human prostate cancer cells via endoplasmic reticulum stress. Anticancer Res 29: 2195-2204, 2009

27. Le MN, Chan JL, Rosenberg SA, Nabatian AS, Merrigan KT, Cohen-Solal KA and Goydos JS: The glutamate release inhibitor Riluzole decreases migration, invasion, and proliferation of melanoma cells. J Invest Dermatol 130: 2240-2249, 2010.

28. Liao S, Ruiz Y, Gulzar H, Yelskaya Z, Ait Taouit L, Houssou M, Jaikaran T, Schvarts Y, Kozlitina K, Basu-Roy K, et al: Osteosarcoma cell proliferation and survival requires mGluR5 receptor activity and is blocked by riluzole. PLoS One 12 : e0171256, 2017.

29. Sperling ST, Aung S, Martin V, Rohde V and Ninkovic M: Riluzole: A potential therapeutic intervention in human brain tumor stem-like cells. Oncotarget 8: 96697-96709, 2017.

30. Yelskaya Z, Carrillo E, Dubisz E, Gulzar H, Morgan D and Mahajan SS: Synergistic inhibition of survival, proliferation, and migration of U87 cells with a combination of LY341495 and iressa. PLoS One 8: e64588, 2013.

31. Zhang C, Yuan XR, Li HY, Zhao ZJ, Liao YW, Wang XY, Su J, Sang SS and Liu Q: Anti-cancer effect of metabotropic glutamate receptor 1 inhibition in human glioma U87 cells: Involvement of PI3K/Akt/mTOR pathway. Cell Physiol Biochem 35: 419-432, 2015.

32. Yip D, Le MN, Chan JL, Lee JH, Mehnert JA, Yudd A, Kempf J, Shih WJ, Chen S and Goydos JS: A phase 0 trial of riluzole in patients with resectable stage III and IV melanoma. Clin Cancer Res 15: 3896-3902, 2009.

33. Mehnert JM, Silk AW, Wen Y, Lee JH, Dudek L, Jeong BS Li J, Schenkel JM, Sadimin E, Kane M, et al: A phase II trial of riluzole, an antagonist of metabotropic glutamate receptor 1 (GRM1) signaling, in patients with advanced melanoma. Pigment Cell Melanoma Res 31: 534-540, 2018.

34. Jia SF, Worth LL and Kleinerman ES: A nude mouse model of human osteosarcoma lung metastases for evaluating new therapeutic strategies. Clin Exp Metastasis 17: 501-506, 1999.

35. Caldorera-Moore M, Guimard N, Shi L and Roy K: Designer nanoparticles: Incorporating size, shape and triggered release into nanoscale drug carriers. Expert Opin Drug Deliv 7: 479-495, 2010.

36. Tomuleasa $\mathrm{C}$, Braicu $\mathrm{C}$, Irimie A, Craciun L and BerindanNeagoe I: Nanopharmacology in translational hematology and oncology. Int J Nanomedicine 9: 3465-3479, 2014.
37. Dadwal A, Baldi A and Kumar Narang R: Nanoparticles as carriers for drug delivery in cancer. Artif Cells Nanomed Biotechnol 46: 295-305, 2018.

38. Sykes EA, Chen J, Zheng G and Chan WC: Investigating the impact of nanoparticle size on active and passive tumor targeting efficiency. ACS Nano 8: 5696-5706, 2014

39. Moghimi SM and Szebeni J: Stealth liposomes and long circulating nanoparticles: Critical issues in pharmacokinetics, opsonization and protein-binding properties. Prog Lipid Res 42: 463-478, 2003

40. Gao Y, Xie J, Chen H, Gu S, Zhao R, Shao J and Jia L: Nanotechnology-Based intelligent drug design for cancer metastasis treatment. Biotechnol Adv 32: 761-777, 2014.

41. Kim PS, Djazayeri S and Zeineldin R: Novel nanotechnology approaches to diagnosis and therapy of ovarian cancer. Gynecol Oncol 120: 393-403, 2011.

42. Champion JA, Katare YK and Mitragotri S: Particle shape: A new design parameter for micro- and nanoscale drug delivery carriers. J Control Release 121: 3-9, 2007.

43. Toy R, Peiris PM, Ghaghada KB and Karathanasis E: Shaping cancer nanomedicine: The effect of particle shape on the in vivo journey of nanoparticles. Nanomedicine (Lond) 9: 121-134, 2014.

44. Truong NP, Whittaker MR, Mak CW and Davis TP: The importance of nanoparticle shape in cancer drug delivery. Expert Opin Drug Deliv 12: 129-142, 2015.

45. Rampersaud S, Fang J, Wei Z, Fabijanic K, Silver S, Jaikaran T, Ruiz Y, Houssou M, Yin Z, Zheng S, et al: The effect of cage shape on nanoparticle-based drug carriers: Anticancer drug release and efficacy via receptor blockade using dextran-coated iron oxide nanocages. Nano Lett 16: 7357-7363, 2016.

46. Oh MH, Yu T, Yu SH, Lim B, Ko KT, Willinger MG, Seo DH, Kim BH, Cho MG, Park JH, et al: Galvanic replacement reactions in metal oxide nanocrystals. Science 340: 964-968, 2013.

47. Liu Y, Chen T, Wu C, Qiu L, Hu R, Li J, Cansiz S, Zhang L, Cui C, $\mathrm{Zhu} \mathrm{G}$, et al: Facile surface functionalization of hydrophobic magnetic nanoparticles. J Am Chem Soc 136: 12552-12555, 2014

48. Ray P, Wu AM and Gambhir SS: Optical bioluminescence and positron emission tomography imaging of a novel fusion reporter gene in tumor xenografts of living mice. Cancer Res 63: $1160-1165,2003$

49. Steckiewicz KP, Barcinska E, Malankowska A, ZauszkiewiczPawlak A, Nowaczyk G, Zaleska-Medynska A and Inkielewicz-Stepniak I: Impact of gold nanoparticles shape on their cytotoxicity against human osteoblast and osteosarcoma in in vitro model. Evaluation of the safety of use and anti-cancer potential. J Mater Sci Mater Med 30: 22, 2019.

50. Groeneveld GJ, Van Kan HJ, Kalmijn S, Veldink JH, Guchelaar HJ, Wokke JH and Van den Berg LH: Riluzole serum concentrations in patients with ALS: Associations with side effects and symptoms. Neurology 61: 1141-1143, 2003.

51. Groeneveld GJ, van Kan HJ, Lie AHL, Guchelaar HJ and van den Berg LH: An association study of riluzole serum concentration and survival and disease progression in patients with ALS. Clin Pharmacol Ther 83: 718-722, 2008.

52. Bondi ML, Craparo EF, Giammona G and Drago F: Brain-targeted solid lipid nanoparticles containing riluzole: Preparation, characterization and biodistribution. Nanomedicine (Lond) 5: 25-32, 2010. International (CC BY-NC-ND 4.0) License. 